\section{Medicamentos excepcionais para doença renal crônica: gastos e perfil de utilização em Minas Gerais, Brasil}

\author{
Dispensing of exceptional drugs for chronic renal \\ failure: expenditures and patients' profile in \\ Minas Gerais State, Brazil
}

\author{
1 Secretaria Estadual de \\ Saúde de Minas Gerais, Belo \\ Horizonte, Brasil. \\ 2 Faculdade de Medicina \\ Universidade Federal de \\ Minas Gerais, Belo Horizonte, \\ Brasil. \\ Correspondência \\ G. D. Silva \\ Secretaria Estadual de Saúde \\ de Minas Gerais. \\ Rua Reynaldo Smith Camargos \\ 264, casa 1, Belo Horizonte, \\ MG 31555-290, Brasil. \\ grazidiassilva@gmail.com
}

\begin{abstract}
In Brazil, medicines for treatment of chronic renal failure are available free of cost from the Unified National Health System (SUS). This study's objectives were to describe government spending on these drugs in Minas Gerais State, Brazil, and the patients' profile, as well as to analyze the factors associated with individual average monthly costs. Spending on medication for chronic renal failure ( $R \$ 41.6$ million, or $U \$ 25$ million) represents a significant portion of total spending on outpatient procedures in the National health System (9.6\%). Most patients are young adult males with arterial hypertension as the main cause of chronic renal failure. Multivariate analysis showed a trend towards lower spending on elderly patients, those with diabetes as the main underlying disease, those using iron hydroxide, and in municipalities with a lower human development index, or HDI $(p<0.05)$. Finally, the study indicated the importance of management tools that allow monitoring the trajectory of individual patients in the health system and support appropriate health policymaking.
\end{abstract}

Exceptional Drugs; Health Expenditures; Single Health System
Grazielle Dias da Silva 1

Francisco de Assis Acúrcio 2 Mariângela Leal Cherchiglia 2 Augusto Afonso Guerra Júnior 1 Eli Iola Gurgel Andrade 2

\section{Introdução}

A doença renal crônica é caracterizada pela perda lenta e irreversível das funções renais. Essa perda pode ser controlada por medicamentos e dieta no estágio inicial da doença, bem como por procedimentos como diálise ou transplante renal quando a função normal dos rins é reduzida em mais de $90 \%$. Um número cada vez maior de brasileiros tem sido atingido por essa enfermidade em virtude do envelhecimento natural da população e do aumento no número de portadores de hipertensão e diabetes mellitus, principais causas da doença renal crônica.

O tratamento tem por objetivos a prevenção, o acompanhamento e a intervenção nas complicações e comorbidades. Uma de suas complicações é a anemia causada, principalmente, pela falta do hormônio eritropoetina, produzido pelos rins, e o seu tratamento consiste na reposição de ferro ou do próprio hormônio ${ }^{1}$. Outra complicação da doença renal crônica é a hiperfosfatemia, caracterizada pelo aumento da concentração plasmática de fosfato, que, em associação com o cálcio, leva à calcificação das artérias coronárias e, consequentemente, à doença cardíaca isquêmica, infarto agudo do miocárdio, parada cardíaca e morte súbita. Essas doenças, juntamente com outras complicações cardiovasculares normalmente observadas em portadores de doença renal crônica, representam, 
aproximadamente, a metade das causas de óbito em pacientes em diálise contínua 2.

No Brasil, os medicamentos disponibilizados pelo Sistema Único de Saúde (SUS) para o tratamento dessas complicações fazem parte do Programa Nacional de Medicamentos de Dispensação em Caráter Excepcional. Inicialmente, o financiamento desses medicamentos era de responsabilidade do Instituto Nacional de Assistência Médica da Previdência Social, e o nome excepcional era utilizado para identificar a modalidade de compra e provisão dos medicamentos de alto custo ${ }^{3,4}$.

Em uma concepção mais atual, consideramse medicamentos de dispensação em caráter excepcional aqueles de alto valor agregado, ou que, pela cronicidade do tratamento, tornam-se excessivamente caros para serem custeados pela população 5. Atualmente, os estados executam e gerenciam o programa, enquanto o financiamento deste fica a cargo do Ministério da Saúde, sob a forma de ressarcimento, e dos recursos dos próprios estados, de forma complementar 3 .

É sabido que o custo do programa tem aumentado de forma progressiva com o passar dos anos e que, em média, $16 \%$ dos gastos com os medicamentos cobertos por ele são destinados aos medicamentos para portadores de doença renal crônica. A tendência de crescimento dos gastos com os medicamentos usualmente denominados de alto custo ou "excepcionais" é confirmada por dados recentes de aumento nas taxas de incidência e prevalência em todo o mundo 1,6,7.

Este estudo teve como objetivo descrever os gastos do Ministério da Saúde, no Brasil e em Minas Gerais, com esses medicamentos, bem como descrever o perfil demográfico e epidemiológico dos usuários destes em Minas Gerais. O estudo analisou, ainda, os fatores associados ao gasto médio mensal individual desses indivíduos com os medicamentos utilizados.

\section{Materiais e métodos}

Para a análise do gasto despendido pelo Ministério da Saúde com medicamentos de alto custo destinados ao tratamento da doença renal crônica no Brasil e em Minas Gerais, foi realizado um estudo valendo-se de dados secundários disponíveis no Sistema de Informações Ambulatoriais (SIA/SUS) do Departamento de Informática do SUS (DATASUS).

Para a análise do perfil e do gasto médio mensal individual dos pacientes que iniciaram diálise em Minas Gerais no período de 2000 a 2004 e que utilizaram medicamentos para o tratamento da doença renal crônica, foi realizado um estudo prospectivo não concorrente com base nos dados disponibilizados na Base Nacional de Dados em TRS (terapia renal substitutiva). Esses medicamentos foram pagos pelo Ministério da Saúde via Autorização de Procedimentos de Alto Custo (APAC/SIA).

Os dados para a análise dos gastos totais com medicamentos utilizados no tratamento da doença renal crônica foram tabulados a partir do sítio da Internet do DATASUS (http://www.data sus.gov.br). Já os dados para análise do perfil dos indivíduos que fizeram uso dos medicamentos para tratamento da doença renal crônica foram extraídos da Base Nacional de Dados em TRS construída com base no SIA/SUS. O detalhamento metodológico de elaboração desta base de dados individualizada, utilizando pareamento probabilístico, está disponível em Cherchiglia et al. 8 e Szuster et al. 9 .

O SIA/SUS é o sistema de informações ambulatoriais que abrange os serviços que não são considerados internações hospitalares e funciona de forma descentralizada, operado por prestadores que informam sua produção ao Ministério da Saúde para a efetivação do pagamento pelos serviços realizados no âmbito do SUS ${ }^{3}$. O sistema é composto pelos módulos de produção Boletim de Produção Ambulatorial e APAC ${ }^{3}$, sendo este último o principal componente utilizado no processo de desenvolvimento da Base Nacional de Dados em TRS. A APAC contém informações sobre todos os procedimentos de alta complexidade/custo, medicamentos de dispensação em caráter excepcional e outros considerados de monitoramento estratégico 10, além de informações clínicas e demográficas dos pacientes. Neste estudo, os códigos de procedimento APAC empregados foram: 3610204; 3614102; 3614101; 3614105; 3614103; 3614104; 3617101.

\section{Tratamento e análise dos dados}

A análise dos gastos totais com medicamentos utilizados no tratamento da doença renal crônica no Brasil e em Minas Gerais considerou o período de 2000 a 2004. Esta análise incluiu dados sobre gastos federais com saúde, relação dos gastos com saúde e Produto Interno Bruto (PIB), gastos por código de procedimento de alto custo e quantidade de procedimentos de alto custo fornecidos.

A análise dos dados relacionados ao perfil dos usuários considerou todos os indivíduos que fizeram diálise no Estado de Minas Gerais e utilizaram um dos seguintes medicamentos: eritropoetina humana recombinante, Sevelamer ou hidróxido de ferro III. Já a análise do gasto 
médio mensal individual foi realizada para indivíduos que cumpriram o primeiro critério e que apresentaram valores de APAC nos primeiros 12 meses de acompanhamento. Indivíduos que não apresentaram valores de APAC por mais de três meses seguidos de acompanhamento foram excluídos da análise de gasto médio mensal individual.

A estatística descritiva dos dados coletados foi realizada por meio de medidas de tendência central e de dispersão, para as variáveis contínuas, e distribuições de freqüências, para as variáveis categóricas. Para a análise dos fatores que influenciaram o gasto médio mensal individual com medicamentos nos primeiros 12 meses de acompanhamento, utilizou-se a análise de regressão linear múltipla, incluindo as seguintes variáveis independentes: idade, causa de doença renal crônica, modalidade inicial de TRS, esquema terapêutico inicial e o índice de desenvolvimento humano municipal (IDH-M) do município de residência do indivíduo no início do tratamento. O IDH-M foi categorizado segundo os quartis de sua distribuição.

Os programas utilizados para possibilitar a organização e análise estatística dos dados foram Microsoft Excel 2000 (Microsoft Corp., Estados Unidos), SPSS 14 (SPSS Inc., Chicago, Estados Unidos) e R for Windows versão 2.5.1 (The R Foundation for Statistical Computing, Viena, Áustria; http://www.r-project.org).

Os valores monetários para os anos entre 2000 e 2004 foram atualizados para dezembro de 2008 pelo Índice Nacional de Preços ao Consumidor Amplo (IPCA), do Instituto Brasileiro de Geografia e Estatística (IBGE).

\section{Aspectos éticos}

Este estudo é parte integrante do projeto Avaliação Econômica e Epidemiológica das Modalidades de Terapias Renais Substitutivas no Brasil, realizado pelo Grupo de Pesquisa em Economia da Saúde da Faculdade de Medicina da Universidade Federal de Minas Gerais (UFMG). O projeto teve parecer favorável da Comissão de Ética em Pesquisa da UFMG (parecer ETIC nº. 397/2004).

\section{Limitações do método}

O estudo analisa apenas os gastos realizados pelo Ministério da Saúde com medicamentos de alto custo. Segundo aponta documento do Conselho Nacional de Secretários da Saúde (CONASS) ${ }^{3}$, os valores repassados pelo Ministério da Saúde para financiamento dos medicamentos de dispensação em caráter excepcional para as Secretarias Estaduais de Saúde não co- bririam a totalidade dos gastos realizados por estas no Programa de Medicamentos de Dispensação em Caráter Excepcional.

Além disso, a análise valeu-se da Base $\mathrm{Na}$ cional de Dados em TRS, construída com dados disponibilizados pelo DATASUS. As bases de dados originais são alimentadas por informações fornecidas pelas Secretarias de Estado da Saúde, e a qualidade destas é dependente dos processos envolvidos no registro e na disponibilização dos dados por sistemas de informação 11. Sendo assim, é possível a existência de dados incorretos, causados por erros na alimentação das bases de dados originais ou por sub-registros, o que pode culminar em sub ou superestimação das análises realizadas.

Vale lembrar, ainda, que a APAC tem validade para três meses de tratamento. Com isso, detectou-se, durante a construção da coorte estudada, que as APAC cuja vigência iniciou-se em novembro e dezembro do ano de 2004 foram registradas no sistema do DATASUS em janeiro de 2005. Portanto, estes registros foram excluídos da coorte no ano de 2004 e, considerando a possibilidade de maior grau de sub-registro para o último ano de acompanhamento, a taxa de incidência foi calculada para o período de 2000 a 2003.

\section{Resultados}

\section{Gastos com medicamentos de alto custo}

Os dados disponibilizados pelo DATASUS indicam que o gasto federal total com saúde representou, em média, $2 \%$ do PIB no período de 2000 a 2004. Já o gasto com medicamentos de alto custo representou, em média, $2 \%$ do gasto federal total com saúde no mesmo período.

Em relação aos gastos com procedimentos ambulatoriais no SUS cobertos pela APAC no mesmo período, os dados apontam para um gasto médio anual de R \$ 9,5 bilhões em todo o Brasil. O gasto com medicamentos de alto custo representou, em média, $9 \%$ do gasto total com esses procedimentos, e os medicamentos utilizados no tratamento da doença renal crônica consumiram, em média, $14 \%$ do valor total gasto com medicamentos de alto custo.

A distribuição dos gastos com medicamentos utilizados no tratamento da doença renal crônica, segundo a região do país, indicou que a Região Sudeste é a que mantém o maior gasto (51\%) no período analisado, seguida das regiões Nordeste (26\%), Sul (14\%), Centro-oeste (6\%) e Norte (3\%). Os estados onde ocorreu maior gasto com esses medicamentos, no mesmo período, foram São Paulo, Rio de Janeiro, Minas Gerais e Ceará. 
Em Minas Gerais, os dados indicam que o gasto federal total com saúde representou, em média, 2\% do PIB no período 2000 a 2004. Já o gasto com medicamentos de alto custo representou, em média, $3 \%$ do gasto federal total com saúde no mesmo período. O gasto médio anual com procedimentos cobertos pela APAC no período estudado foi de aproximadamente $\mathrm{R} \$ 962,5 \mathrm{mi}-$ lhões, e o gasto com medicamentos de alto custo representou, em média, $10 \%$ do gasto total com esses procedimentos no estado. Em adição, os medicamentos de alto custo utilizados no tratamento da doença renal crônica consumiram, em média, $10 \%$ do gasto com estes medicamentos (Tabela 1). O gasto per capita por macrorregião de saúde de Minas Gerais para os medicamentos em análise variou de $\mathrm{R} \$ 0,21$ (Jequitinhonha) a $\mathrm{R} \$ 6,27$ (Centro), com média de $\mathrm{R} \$ 0,93$.

O medicamento de alto custo utilizado no tratamento da doença renal crônica de maior gasto no SUS foi a eritropoetina humana recombinante, tanto no Brasil ( $88 \%$ dos gastos), quanto em Minas Gerais (92\% dos gastos). O medicamento cloridrato de Sevelamer é o segundo de maior gasto no Brasil dentre os medicamentos citados (8\% dos gastos). Em Minas Gerais, o medicamento que ocupa este lugar é o hidróxido de ferro III (6\% dos gastos). O gasto com esses medicamentos apresentou uma variação percentual média anual negativa de 9,5\% em Minas Gerais, no período de 2000 a 2004, descontada a inflação para o período.
Perfil dos usuários do SUS de Minas Gerais que utilizaram medicamentos para tratamento da doença renal crônica

A Base Nacional de Dados em TRS permitiu a identificação de 10.603 indivíduos residentes em Minas Gerais no momento do primeiro registro de APAC $\left(\mathrm{t}^{0}\right)$ portadores de doença renal crônica que utilizaram, no mínimo, um dos medicamentos analisados no período estudado.

Dos usuários identificados, $57 \%$ eram do sexo masculino, com idade média de 47 anos $( \pm 17$ anos). A principal causa de doença renal crônica identificada foi hipertensão arterial/doenças cardiovasculares (22\%), seguida de glomerulonefrites (14\%) e diabetes mellitus (13\%). É importante ressaltar o elevado percentual de causas indeterminadas de doença renal crônica (46\%). Observou-se, ainda, que a grande maioria dos usuários iniciou o tratamento de substituição renal na modalidade de hemodiálise $(90 \%)$, mas apenas $6 \%$ iniciaram em alguma modalidade de diálise peritoneal. A distribuição dos usuários por região de residência no to apontou que a maior parte residia nas macrorregiões de saúde Centro (37\%), Sul (14\%) e Sudeste (9\%) (Tabela 2).

A taxa média de incidência de terapia renal substitutiva observada em Minas Gerais, para o período de 2000 a 2003, foi 76 pessoas por milhão de habitantes e decresceu em média $4 \%$ ao ano na coorte estudada (Tabela 3 ).

Tabela 1

Gastos realizados pelo Ministério da Saúde com procedimentos de alto custo no Estado de Minas Gerais, Brasil, 2000 a 2004.

\begin{tabular}{|c|c|c|c|c|c|}
\hline \multirow[t]{2}{*}{ Ano } & \multicolumn{5}{|c|}{ Total dos gastos (em Reais) } \\
\hline & SIA/SUS & $\begin{array}{c}\text { Medicamentos de } \\
\text { alto custo }\end{array}$ & $\begin{array}{c}\text { SIA/SUS/ } \\
\text { Medicamentos de } \\
\text { alto custo (\%) }\end{array}$ & $\begin{array}{c}\text { Medicamentos } \\
\text { de alto custo } \\
\text { no tratamento } \\
\text { de doença renal } \\
\text { crônica }\end{array}$ & $\begin{array}{l}\text { Medicamentos } \\
\text { de alto custo } \\
\text { no tratamento } \\
\text { de doença } \\
\text { renal crônica/ } \\
\text { Medicamento de } \\
\text { alto custo (\%) }\end{array}$ \\
\hline 2000 & $1.043 .819 .460,81$ & $80.135 .102,03$ & 7,68 & $9.023 .807,09$ & 11,26 \\
\hline 2001 & $773.913 .044,37$ & $84.800 .915,07$ & 10,96 & $11.095 .850,97$ & 13,08 \\
\hline 2002 & $818.157 .111,38$ & $86.567 .669,23$ & 10,58 & $8.424 .145,68$ & 9,73 \\
\hline 2003 & $853.204 .597,91$ & $79.915 .390,54$ & 9,37 & $7.044 .774,70$ & 8,82 \\
\hline 2004 & $863.240 .711,76$ & $84.822 .591,18$ & 9,83 & $6.043 .316,32$ & 7,12 \\
\hline Média & $870.466 .985,25$ & $83.248 .333,61$ & 9,68 & $8.326 .378,95$ & 10,0 \\
\hline $2000-2004$ & 4.352.334.926,23 & $416.241 .668,06$ & 9,56 & $41.631 .894,76$ & 10,00 \\
\hline
\end{tabular}

SIA/SUS: Sistema de Informações Ambulatoriais do Departamento de Informática do SUS (DATASUS). 
Características dos usuários do Sistema Único de Saúde (SUS) que utilizaram medicamentos de alto custo para tratamento da doença renal crônica em Minas Gerais, Brasil, 2000 a 2004.

\begin{tabular}{|c|c|c|}
\hline Características dos usuários & $\mathrm{n}$ & $\%$ \\
\hline \multicolumn{3}{|l|}{ Idade (anos) à entrada em TRS [média (DP)] } \\
\hline 47 anos (DP \pm 17 ) & 10.586 & \\
\hline Missing & 17 & \\
\hline \multicolumn{3}{|l|}{ Sexo } \\
\hline Feminino & 4.599 & 43,4 \\
\hline Masculino & 6.004 & 56,6 \\
\hline Total & 10.603 & 100,0 \\
\hline \multicolumn{3}{|l|}{ Causa de doença renal crônica no to } \\
\hline Hipertensão/Doenças cardiovasculares & 2.309 & 21,8 \\
\hline Glomerulonefrites & 1.478 & 13,9 \\
\hline Diabetes mellitus & 1.326 & 12,5 \\
\hline Indeterminada & 4.860 & 45,8 \\
\hline Outras & 630 & 6,0 \\
\hline Total & 10.603 & 100,0 \\
\hline \multicolumn{3}{|l|}{ Modalidade de TRS no to } \\
\hline Hemodiálise & 9561 & 90,2 \\
\hline Diálise peritoneal ambulatorial contínua & 525 & 5,0 \\
\hline Transplante renal & 384 & 3,6 \\
\hline Diálise peritoneal automática & 86 & 0,8 \\
\hline Indeterminada & 47 & 0,4 \\
\hline Total & 10.603 & 100,0 \\
\hline \multicolumn{3}{|l|}{ Macrorregião de residência no to } \\
\hline Centro & 3.931 & 37,2 \\
\hline Sul & 1.422 & 13,4 \\
\hline Sudeste & 975 & 9,2 \\
\hline Triângulo do Norte & 720 & 6,8 \\
\hline Leste & 673 & 6,4 \\
\hline Oeste & 627 & 5,9 \\
\hline Norte de Minas & 552 & 5,2 \\
\hline Centro Sul & 448 & 4,2 \\
\hline Leste do Sul & 418 & 4,0 \\
\hline Triângulo do Sul & 320 & 3,0 \\
\hline Noroeste & 236 & 2,2 \\
\hline Nordeste & 226 & 2,1 \\
\hline Jequitinhonha & 55 & 0,5 \\
\hline Total & 10.603 & 100,0 \\
\hline
\end{tabular}

DP: desvio-padrão; t0: mês de início do tratamento da doença renal crônica; TRS: terapia renal substitutiva

Análise do gasto médio mensal individual com medicamentos nos 12 primeiros meses de acompanhamento

Para a análise do gasto médio mensal individual nos primeiros 12 meses de acompanhamento foram considerados 10.006 indivíduos. Foram excluídos desta análise 597, que não apresentaram registro de gastos nos três primeiros meses de acompanhamento.
As variáveis incluídas no modelo multivariado final explicaram $23 \%$ da variação observada no gasto médio mensal individual. Ao controlar a idade pelas demais variáveis, observou-se que o aumento da idade leva a um menor gasto ( $p<0,001)$. Em relação à causa de doença renal crônica, o modelo apontou para tendência de maior gasto entre indivíduos com diagnóstico de hipertensão arterial/doenças cardiovasculares ( $\mathrm{p}<0,05)$ e glomerulonefrites $(\mathrm{p}<0,001)$ e para 
Taxa de incidência de doença renal crônica na coorte de usuários do Sistema Único de Saúde (SUS) que utilizaram medicamentos de alto custo em Minas Gerais, Brasil, 2000 a 2004.

\begin{tabular}{lcccc}
\hline Ano & $\begin{array}{c}\text { Indivíduos com doença } \\
\text { renal crônica em Minas } \\
\text { Gerais (n) }\end{array}$ & $\begin{array}{c}\text { População em Minas } \\
\text { Gerais }\end{array}$ & $\begin{array}{c}\text { Taxa de incidência por } \\
\text { milhão de habitantes * }\end{array}$ & Variação (\%) \\
\hline 2000 & 1.394 & 17.492 .296 & 79,69 & - \\
2001 & 1.365 & 18.127 .096 & 75,30 & $-5,5$ \\
2002 & 1.443 & 18.343 .517 & 78,67 & 4,5 \\
2003 & 1.312 & 18.553 .312 & 70,72 & $-10,1$ \\
Média & 1.379 & 18.129 .055 & 76,04 & $-3,7$ \\
\hline
\end{tabular}

* Taxas calculadas para a coorte em estudo.

tendência de menor gasto entre aqueles com causa indeterminada de doença renal crônica ( $\mathrm{p}<0,001$ ), quando comparados a indivíduos com diagnóstico de diabetes mellitus (Tabela 4).

No que diz respeito à modalidade de início de tratamento, observou-se a tendência de maior gasto entre indivíduos que utilizaram a diálise peritoneal e de menor gasto entre os que iniciaram a TRS em transplante renal ou que não tiveram uma modalidade determinada, quando comparados a indivíduos que utilizaram hemodiálise ( $p<0,001)$. Já a análise do esquema terapêutico inicial indicou um gasto menor entre indivíduos que utilizaram esquemas terapêuticos incluindo hidróxido de ferro como medicamento de primeira escolha no tratamento da anemia na doença renal crônica, quando comparados àqueles em esquemas terapêuticos que incluíam eritropoetina humana como medicamento de primeira escolha $(\mathrm{p}<0,001)$ (Tabela 4$)$.

$\mathrm{O}$ IDH-M mostrou influência no gasto médio mensal individual. Quanto menor o IDH-M do município de residência no início do tratamento, menor o gasto observado ( $\mathrm{p}<0,01$ ) (Tabela 4).

\section{Discussão}

\section{Gastos com medicamentos de alto custo}

O gasto referente à saúde é motivo de debate em vários países do mundo. No Brasil, o gasto federal total com saúde representou, em média, 1,9\% em relação ao PIB nacional, e o gasto com medicamentos de alto custo representou, em média, $2,2 \%$ em relação ao gasto federal total com saúde no período analisado. $\mathrm{O}$ perfil desses gastos não foi diferente para o Estado de Minas Gerais. A participação do gasto federal com saúde em relação ao PIB, no Brasil e em Minas Gerais, en- contra-se muito abaixo do observado para outros países, como, por exemplo, Itália, Alemanha, Canadá, Japão, Estados Unidos, França e Bélgica, onde variou de 7,9\% no Japão a 14,6\% nos Estados Unidos 12,13,14,15,16,17.

As diferenças institucionais de acesso a políticas de bem-estar social constituem-se em pano de fundo para que se compreendam as diferenças de gastos e organização desses sistemas de saúde. Contudo, também se pode compreender a variação no nível de gasto com saúde em cada país por características demográficas, étnicas, culturais, econômicas, bem como por diferenças apresentadas em incentivos governamentais para aumento da qualidade e da eficiência dos serviços de saúde prestados 18 .

Em relação aos gastos com procedimentos ambulatoriais no SUS cobertos pela APAC no mesmo período, os dados demonstram que a despesa com medicamentos utilizados no tratamento da doença renal crônica consome uma parcela significativa do gasto total com tais procedimentos, tanto no Brasil, quanto em Minas Gerais.

Na Itália, o gasto com o tratamento da doença renal crônica consome, aproximadamente, $2 \%$ do gasto total com saúde, porém o gasto com medicamentos está incluído no valor das sessões de diálise e, com exceção da eritropoetina humana, estão sujeitos a co-pagamento por parte dos pacientes 17. Já em países como Alemanha, Canadá e Austrália, todos os medicamentos são custeados pelo governo com co-participação dos pacientes 15,16,19.

Em países como Japão, França, Bélgica, Argentina, Cuba, Porto Rico, Uruguai e Venezuela, os custos de medicamentos para o tratamento da doença renal crônica são totalmente cobertos pelo sistema de saúde, assim como ocorre no Brasil 12,13,14,20. A participação dos gastos com o 
Análise multivariada do gasto médio mensal individual dos usuários do Sistema Único de Saúde (SUS) que utilizaram algum medicamento de alto custo para o tratamento da doença renal crônica em Minas Gerais, Brasil, 2000 a 2004.

\begin{tabular}{|c|c|c|c|}
\hline Variáveis explicativas & $\beta$ & $\begin{array}{c}\text { Erro- } \\
\text { padrão }\end{array}$ & Valor de $p$ \\
\hline Intercepto & 2061,944 & 22,871 & $<0,001$ \\
\hline Idade à entrada em TRS & $-3,67$ & 0,296 & $<0,001$ \\
\hline \multicolumn{4}{|c|}{ Causa de doença renal crônica no to (categoria de referência: diabetes mellitus) } \\
\hline Hipertensão arterial/Doenças cardiovasculares & 39,67 & 17,195 & $<0,05$ \\
\hline Glomerulonefrites & 76,086 & 19,278 & $<0,001$ \\
\hline Causa indeterminada & $-62,246$ & 15,646 & $<0,001$ \\
\hline Outras causas & $-32,397$ & 27,179 & $>0,05$ \\
\hline \multicolumn{4}{|c|}{ Modalidade de TRS no to (categoria de referência: hemodiálise) } \\
\hline Diálise peritoneal & 652,508 & 20,684 & $<0,001$ \\
\hline Transplante renal & $-1023,546$ & 29,754 & $<0,001$ \\
\hline Indeterminada & $-1226,511$ & 87,293 & $<0,001$ \\
\hline \multicolumn{4}{|c|}{ Esquema terapêutico inicial to (categoria de referência: eritropoetina humana) } \\
\hline Hidróxido de ferro & $-63,799$ & 10,987 & $<0,001$ \\
\hline Sevelamer & $-3,069$ & 242,509 & $>0,05$ \\
\hline Outros & 14,113 & 13,899 & $>0,05$ \\
\hline \multicolumn{4}{|c|}{ IDH-M no to (categoria de referência: $0,00-0,76$ - 1ㅇ quartil) } \\
\hline $0,77-0,80$ (2o quartil) & $-35,084$ & 13,18 & $<0,01$ \\
\hline $0,81-0,84$ (3o quartil) & $-2,845$ & 11,841 & $>0,05$ \\
\hline Acima de 0,85 (4o quartil) & 63,703 & 57,62 & $>0,05$ \\
\hline
\end{tabular}

Nota: variável dependente - gasto médio mensal individual nos primeiros 12 meses de acompanhamento $(R 2=23,2 \%)$.

IDH-M: índice de desenvolvimento humano municipal; to: mês de início do tratamento da doença renal crônica; TRS: terapia renal substitutiva.

tratamento da doença renal crônica no gasto total com saúde variou de $1,3 \%$, para a França, a 3,7\%, para o Japão; os custos dos medicamentos estão incluídos nos custos totais da diálise 12,13,14.

O medicamento utilizado no tratamento da doença renal crônica de maior gasto observado foi a eritropoetina humana recombinante, seja no Brasil, seja em Minas Gerais. Esse fato pode ser explicado pelo alto percentual de pacientes em uso deste medicamento no país (86\%), seguindo a tendência apresentada por países como Canadá e Austrália, onde a prevalência de pacientes com doença renal crônica em uso de eritropoetina é de $91 \%$ e $86 \%$, respectivamente 16,19 .

Em Minas Gerais, a estrutura de gastos com medicamentos de alto custo para tratamento da doença renal crônica, ilustrada na Tabela 1, chama a atenção pela variação observada. A hipótese que poderia explicar a variação percentual negativa nos gastos observada no período estudado diz respeito à grande diferença no valor do dólar de 2000 a 2004, chegando a ter uma variação percentual de $-8,1 \%$ a $4,1 \%$ em 2002 , ano em que se inicia a diminuição no valor gasto com os medicamentos em estudo. A eritropoetina humana passou a ser produzida no Brasil apenas em 2006, sendo totalmente importada no período correspondente à coorte estudada, o que explica a influência da variação do dólar nos valores gastos pelo Estado de Minas Gerais com esta classe de medicamentos. Dados apresentados pela Federação Brasileira da Indústria Farmacêutica (2004) parecem confirmar essa tendência ao indicarem uma queda no preçofábrica de medicamentos de $24 \%$ para o Brasil e de $15 \%$ no mercado mundial 21 .

\section{Perfil dos usuários}

A Base Nacional de Dados em TRS permitiu a caracterização dos usuários de medicamentos em Minas Gerais. A maioria é do sexo masculino, adultos jovens e teve como causas principais de doença renal crônica a hipertensão arterial/ doenças cardiovasculares, glomerulonefrites e diabetes mellitus. É preocupante o elevado percentual de causas indeterminadas dessa doença, 
conforme observado no estudo, o que pode estar relacionado à qualidade do registro ou da atenção oferecida.

Outras investigações desenvolvidas no Brasil apontam para o mesmo perfil apresentado para portadores de doença renal crônica em Minas Gerais 22,23. Segundo Sesso 24, apesar de a validade dos diagnósticos de causa de doença renal crônica ser questionável em virtude da não comprovação histológica na maioria dos casos, supõe-se que a hipertensão arterial permaneça como principal causa da doença no país por sua alta prevalência e pelo número de indivíduos que não recebem tratamento adequado.

Dados da Sociedade Brasileira de Nefrologia (SBN), datados de 2007, indicam, igualmente, alta prevalência de pacientes com diabetes mellitus (26\%) 25. Esse diagnóstico foi observado como principal causa de doença renal crônica em Porto Rico, México, Bolívia, Chile, Colômbia, Argentina e Guatemala 20 e tem levado ao aumento da incidência da doença em vários outros países 12,13,14,15,16. Os dados da SBN apontam, ainda, para uma maior freqüência de pacientes com idade entre 19 e 64 anos em TRS no Brasil 25, diferindo do encontrado em países desenvolvidos como Itália, Alemanha, Canadá, Bélgica e Austrália, cuja idade de início de tratamento varia de 62 a 69 anos 12,13,15,16,17,19. Acredita-se que a entrada tardia em TRS em países desenvolvidos seja fruto tanto do envelhecimento natural da população, como da capacidade dos serviços de atenção primária em detectar de forma precoce a doença $12,13,14,15,16,17,19$

Em Minas Gerais, a taxa de incidência de TRS observada para o período de 2000 a 2003 foi, em média, 76 pessoas por milhão de habitantes e apresentou uma variação percentual média negativa na coorte estudada. No Brasil, a taxa de incidência média é de 181 pessoas por milhão 25 e, assim como em Minas Gerais, também apresenta uma redução no ritmo de entrada de pacientes em TRS. A principal hipótese que poderia explicar este fato seria a da estagnação no número de unidade de diálise nos últimos anos, diminuindo a capacidade do sistema de absorver os novos casos de doença renal crônica diagnosticados no país 25. Torna-se relevante ressaltar que o Brasil tem investido de forma significativa na estruturação e qualificação da atenção primária em saúde; contudo, supõe-se que uma significativa parcela da população brasileira com doença renal crônica ainda não tenha iniciado a TRS, uma vez que estudos têm apontado para o aumento da prevalência de hipertensão arterial e diabetes mellitus no país 23,24,26,27,28,29.

Na América Latina, a incidência de doença renal crônica é crescente, e os países em que se observam as maiores taxas de incidência são Porto Rico, México, Chile e Uruguai 20. Nestes, igualmente, o número de unidades de diálise é insuficiente para cobrir a demanda por TRS 20 . Nas nações desenvolvidas, a incidência da doença varia de 97 pessoas por milhão na Austrália a 340 pessoas por milhão nos Estados Unidos 18 . No Canadá, Japão, França, Bélgica e Austrália há um crescimento elevado das taxas de incidência, seja pelo envelhecimento acelerado da população nesses países, seja pela alta prevalência das doenças de base da doença renal crônica como diabetes mellitus 12,13,14,16,19. Já na Itália, a incidência da enfermidade tem uma das menores taxas de crescimento entre os países europeus, e isso se deve, principalmente, a ações de promoção e prevenção de doenças crônicas desenvolvidas nesse país 17 .

O presente estudo identificou, também, que a maioria dos usuários em Minas Gerais iniciou o tratamento de substituição renal na modalidade de hemodiálise. Este dado não difere do observado para o Brasil 25 e outros países da América Latina 20, além de países desenvolvidos como Itália, Alemanha, Canadá, Japão, França, Bélgica e Austrália. Nestes últimos a explicação para tal fato encontra-se na equivalência do incentivo financeiro governamental para a modalidade de diálise peritoneal 12,13,14,15,16,17,19. O mesmo não foi observado para El Salvador, Guatemala, México, Nicarágua, Paraguai e República Dominicana, onde a maioria dos pacientes realiza TRS na modalidade de diálise peritoneal 20.

\section{Gasto médio mensal individual}

A análise multivariada indicou uma tendência de menor gasto para indivíduos que iniciaram a TRS na modalidade de transplante renal. Em um estudo realizado nos Estados de São Paulo, Rio de Janeiro, Paraná e Minas Gerais, observou-se que a maior prevalência de anemia se dá em estágios mais avançados da doença renal 22 . Esse fato poderia explicar o menor gasto com medicamentos apresentado por pacientes que iniciaram a TRS com transplante renal, uma vez que este procedimento restaura as funções renais na maioria dos casos 30,31

Segundo Dor et al. 18, a escolha da modalidade de TRS talvez seja o principal fator para a variação observada em resultados clínicos, processos e custos. Em países desenvolvidos, como Bélgica e Itália, a maior despesa por paciente no primeiro ano do transplante é com o procedimento hospitalar, e não com os medicamentos, o que reforça a tendência de menor gasto individual nos primeiros 12 meses de acompanhamento para esta modalidade 12,17 . Por outro lado, no Ja- 
pão, não se observam diferenças significativas no gasto com medicamentos entre as modalidades de TRS, já que as taxas de reembolso não diferem entre si no país 14. Em estudos internacionais, as altas taxas de transplante renal foram associadas às políticas de incentivo à doação de órgãos e de minimização de custo aos pacientes realizadas por diferentes países desenvolvidos 12,15,16,17,18.

Ainda em relação às modalidades, observouse que a categoria de modalidade indeterminada apresentou menor gasto quando comparada à hemodiálise. Tal observação pode ser explicada pelo fato de que os indivíduos não permanecem em TRS, uma vez que sua presença no banco de dados está relacionada à realização de procedimentos como preparação de fístula arteriovenosa, que não necessariamente levariam à utilização de medicamentos.

A análise indicou, ainda, que indivíduos que têm como diagnóstico principal hipertensão arterial/doenças cardiovasculares e glomerulonefrites tendem a um maior gasto do que aqueles que têm como diagnóstico principal o diabetes mellitus. Neste grupo, há um maior percentual de pacientes que iniciaram TRS com idade mais avançada, além de estes adotarem esquema terapêutico incluindo hidróxido de ferro como medicamento de primeira escolha para o tratamento da anemia na doença renal crônica, quando comparados a indivíduos com outras causas dessa enfermidade $(\mathrm{p}<0,0001)$. Segundo Manley 32 , indivíduos com diabetes mellitus devidamente acompanhados na atenção primária têm entrada tardia em TRS e iniciam tratamento em melhores condições clínicas, o que poderia ser um fator para explicar o menor gasto com medicamentos. Em um estudo realizado por Corniali 26 no Estado da Bahia, observou-se maior proporção de pacientes com doença renal cadastrados no HiperDia (Programa Nacional de Cadastramento de Hipertensos e Diabéticos) em idades mais avançadas, indicando melhor acompanhamento destes na atenção primária.

\section{Considerações finais}

Segundo Sólon et al. 33 (p. 22), é no papel predominante do SUS na alta complexidade que o sistema "alcança total consistência aos princípios que lhes dão sustentação, como a universalidade do acesso e a integralidade da atenção". Ainda segundo esses autores, as principais explicações para tal fato seriam a ausência de muitos procedimentos de alto custo nos serviços dos planos de saúde, a provisão pelo SUS de procedimentos e medicamentos que não são acessíveis financeiramente à maioria da população e a melhor percepção de qualidade do sistema de saúde por aqueles que recebem serviços de alta densidade tecnológica.

O caso das TRS não contradiz o exposto por Sólon et al. 33, uma vez que, de certa forma, atende aos critérios explicitados. Entretanto, os indivíduos, em sua maioria, passam a usufruir dos benefícios oferecidos tardiamente, quando já se tornaram portadores de doenças complexas e/ ou de alto custo. Além disso, o presente estudo apontou algumas contradições em relação a conceitos já estabelecidos na literatura sobre TRS no que diz respeito ao exercício da universalidade do acesso e da integralidade da atenção à saúde.

Em relação ao acesso universal aos tratamentos de alto custo, os dados analisados para o caso das TRS na coorte de Minas Gerais indicam que o transplante renal como terapia inicial teve maior proporção entre indivíduos que residiam em municípios de maior IDH-M. Ainda sob este aspecto, o estudo apontou um menor gasto com medicamentos para indivíduos que residiam em municípios de menor IDH-M, podendo indicar um acesso mais restrito para os residentes em municípios menos desenvolvidos.

No que diz respeito à integralidade, o estudo da coorte de Minas Gerais aponta para melhor acompanhamento na atenção primária para indivíduos com diagnóstico de diabetes, já que estes iniciam a TRS em idade mais avançada e apresentam menor gasto com medicamentos, indicando melhores condições clínicas no início do tratamento. Todavia, observou-se um número elevado de pacientes que iniciaram a TRS em idade produtiva, ao contrário do encontrado em países que priorizam as políticas de saúde voltadas para a atenção primária, principalmente no que diz respeito à prevenção e à promoção da saúde.

Ressalte-se, ainda, que a atenção integral explicitada na lei que rege o SUS inclui a assistência farmacêutica. No cenário internacional, no caso das TRS, a terapia medicamentosa normalmente está incluída no procedimento de diálise, e o paciente tem a opção de receber o medicamento na unidade em que é acompanhado. No Brasil, o paciente realiza diálise em uma unidade especializada, recebe o medicamento em uma farmácia do SUS e é transplantado em um hospital, geralmente por um nefrologista diferente daquele que o acompanhou na diálise, demonstrando uma fragmentação do cuidado no âmbito das TRS.

Também no que tange à assistência farmacêutica, é sempre importante voltar à temática da produção nacional de medicamentos. Apesar dos avanços alcançados nos últimos anos, como 
o investimento na aquisição de tecnologias para produção de eritropoetina por laboratórios oficiais desde 2006, no Brasil, ao contrário do que ocorre em países desenvolvidos, mantém-se o foco na produção de medicamentos para atendimento da atenção primária em saúde. Estes medicamentos geralmente têm um baixo valor agregado e grande concorrência no mercado farmacêutico, o que facilita a sua aquisição por todos os níveis de governo. Portanto, uma política de investimento em desenvolvimento e produção de medicamentos de alto custo no país poderia viabilizar maior cobertura assistencial.

\section{Resumo}

No Brasil, os medicamentos para o tratamento da doença renal crônica são disponibilizados gratuitamente pelo Sistema Único de Saúde (SUS). Este estudo teve como objetivos descrever os gastos públicos com esses medicamentos em Minas Gerais, Brasil, e o perfil dos usuários; objetivou, também, analisar os fatores associados ao gasto médio mensal individual. Observou-se que o gasto total com os medicamentos estudados ( $R \$$ 41,6 milhões) representa uma parcela significativa do gasto total com procedimentos ambulatoriais no SUS (9,6\%). A maioria dos usuários é do sexo masculino, adultos jovens e teve como causa principal de doença renal crônica a hipertensão arterial. A análise multivariada indicou tendência de menor gasto entre indivíduos que eram mais idosos, que tinham como causa principal da doença o diabetes, que fizeram uso de hidróxido de ferro e que residiam em municípios de menor IDH-M $(p<0,05)$. Finalmente, o estudo indicou a importância de ferramentas gerenciais que permitam visualizar a trajetória dos pacientes no sistema de saúde, as quais sejam capazes de subsidiar o processo de formulação de políticas de saúde.

Medicamentos Excepcionais; Gastos em Saúde; Sistema Único de Saúde
Finalmente, este estudo possibilitou entender como se dá a construção das políticas de saúde sob o ponto de vista das TRS, no Brasil e em Minas Gerais, por meio da interpretação de dados demográficos, epidemiológicos e econômicos obtidos de bases de dados de faturamento. As informações aqui apresentadas apontam para a necessidade de desenvolvimento de ferramentas gerenciais que permitam visualizar a trajetória dos indivíduos no sistema de saúde e que sejam capazes de ajudar na análise situacional, subsidiando o processo de formulação de políticas de saúde no país.

\section{Colaboradores}

G. D. Silva e A. A. Guerra Júnior participaram da elaboração do artigo e do banco de dados, análise dos dados e revisão final do texto. E. I. G. Andrade e F. A. Acúrcio colaboraram na elaboração do artigo, análise de dados e revisão final do texto. M. L. Cherchiglia contribuiu na elaboração do artigo, fornecimento e análise de dados e revisão final do texto.

\section{Agradecimentos}

Ao Fundo Nacional de Saúde, pelo financiamento. 


\section{Referências}

1. Sociedade Brasileira de Nefrologia. Diretrizes brasileiras de doença renal crônica. São Paulo: Sociedade Brasileira de Nefrologia; 2004.

2. Secretaria de Assistência à Saúde, Minas Gerais. Protocolos clínicos e diretrizes terapêuticas: medicamentos excepcionais. http://www.opas.org. br/medicamentos/docs/pcdt/05_protocolos.pdf (acessado em Ago/2004).

3. Conselho Nacional de Secretários de Saúde. Para entender a gestão do Programa de Medicamentos de Dispensação em Caráter Excepcional. Brasília: Conselho Nacional de Secretários de Saúde; 2004. (CONASS Documenta, 3).

4. Silva RCS. Medicamentos excepcionais no âmbito da assistência farmacêutica no Brasil [Dissertação de Mestrado]. Rio de Janeiro: Escola Nacional de Saúde Pública, Fundação Oswaldo Cruz; 2000.

5. Secretaria de Políticas Públicas, Ministério da Saúde. Assistência farmacêutica na atenção básica: instruções técnicas para sua organização. Brasília: Ministério da Saúde; 2001.

6. Cusumano AM, Di Gioia C, Hermida O, Lavorato C; Latin American Registry of Dialysis and Renal Transplantation. The Latin American Dialysis and Renal Transplantation Registry Annual Report 2002. Kidney Int Suppl 2005; (97):S46-52.

7. Harrison TR, Fauci AS. Harrison medicina interna. 15a Ed. Rio de Janeiro: McGraw-Hill; 2002.

8. Cherchiglia ML, Guerra Júnior AA, Andrade EIG, Machado CJ, Acúrcio FA, Meira Júnior W, et al. A construção da base de dados nacional em terapia renal substitutiva (TRS) centrada no indivíduo: aplicação do método de linkage determinístico-probabilístico. Rev Bras Estud Popul 2007; 24:163-7.

9. Szuster DAC, Silva GM, Andrade ELG, Acúrcio FA, Caiaffa WT, Gomes IC, et al. Potencialidades do uso de bancos de dados para informação em saúde: o caso das terapias renais substitutivas (TRS) - morbidade e mortalidade dos pacientes em TRS. Rev Méd Minas Gerais 2009; 19:317-24.

10. Teixeira OG. Acesso aos procedimentos de alta complexidade no âmbito do SUS em Belo Horizonte/MG: o caso da utilização da quimioterapia e radioterapia por pacientes portadoras de câncer de mama residentes em Belo Horizonte, nos anos de 2000 e 2001 [Dissertação de Mestrado]. Belo Horizonte: Faculdade de Medicina, Universidade Federal de Minas Gerais; 2003.

11. Moraes IHS, Santos SRFR. Informações para a gestão do SUS: necessidades e perspecivas. Inf Epidemiol SUS 2001; 10:49-56.

12. Biesen WV, Lameire N, Peeters P, Vanholder R. Belgium's mixed private/public health care system and its impact on the cost of end-stage renal disease. Int J Health Care Finance Econ 2007; 7:133-48.

13. Durand-Zaleski I, Combe C, Lang P. International study of health care organization and financing for end-stage renal disease in France. Int J Health Care Finance Econ 2007; 7:171-83.

14. Fukuhara S, Yamazaki C, Hayashino Y, Higashi T, Eichleay MA, Akiba T, et al. The organization and funding of end-stage renal disease treatment in Japan. Int J Health Care Finance Econ 2007; 7:217-31.
15. Kleophas W, Reichel H. International study of health care organization and financing: development of renal replacement therapy in Germany. Int J Health Care Finance Econ 2007; 7:185-200.

16. Manns BJ, Mendelssohn DC, Taub KJ. The economics of end-stage renal disease care in Canada: incentives and impact on delivery of care. Int J Health Care Finance Econ 2007; 7:149-69.

17. Pontoriero G, Pozzoni P, Vecchio LD, Locatelli FH. International study of health care organization and financing for renal replacement therapy in Italy: an evolving reality. Int J Health Care Finance Econ 2007; 7:201-15.

18. Dor A, Pauly MV, Eichleay MA, Held PJ. End-stage renal disease and economics incentives: the International Study of Health Care Organization and Financing (ISHCOF). Int J Health Care Finance Econ 2007; 7:73-111.

19. Harris A. The organization and funding of the treatment of end-stage renal disease in Australia. Int J Health Care Finance Econ 2007; 7:113-32.

20. Cusumano A, Garcia-Garcia G, Di Gioia C, Hermida O, Lavorato C, Carreño CA, et al. End-stage renal disease and its treatment in Latin American in the twenty-first century. Ren Fail 2006; 28:631-7.

21. Ohana EF. Comparativo internacional de preços de produtos farmacêuticos em 2004. São Paulo: Federação Brasileira da Indústria Farmacêutica; 2005.

22. Canziani MEF, Bastos MG, Bregman R, Pecoits Filho R, Tomiyama C, Draibe SA, et al. Deficiência de ferro e anemia na doença renal crônica. J Bras Nefrol 2006; XXVIII:86-90.

23. Romão Jr. JE, Haiashi AR, Elias RM, Luders C, Ferraboli R, Castro MCM, et al. Alterações de cálcio e fósforo séricos e hiperparatireoidismo na insuficiência renal crônica incidente. J Bras Nefrol 2004; XXVI:6-11.

24. Sesso R. Epidemiologia da doença renal crônica. ftp://ftp.cve.saude.sp.gov.br/doc_tec/cronicas/ irc_prevprof.pdf (acessado em Fev/2008).

25. Sesso R, Lopes AA, Thomé FS, Bevilacqua JL, Romão Jr. JE, Lugon J. Resultados do Censo de Diálise da SBN, 2007. J Bras Nefrol 2007; XXIX:197-202.

26. Corniali AR. Planejamento da oferta de serviços de terapia renal substitutiva na Bahia [Dissertação de Mestrado]. Salvador: Universidade Federal da Bahia; 2005.

27. Leite IC, Schramm JMA, Gadelha AMJ, Valente JG, Campos MR, Portela MC, et al. Comparação das informações sobre as prevalências de doenças crônicas obtidas pelo suplemento saúde da PNAD/98 e as estimativas pelo estudo Carga de Doença no Brasil. Ciênc Saúde Coletiva 2002; 7:733-41.

28. Romão Jr. JE, Pinto SWL, Canziani ME, Praxedes JN, Santello JL, Moreira JCM. Censo SBN 2002: informações epidemiológicas das unidades de diálise do Brasil. J Bras Nefrol 2003; XXV:188-99.

29. Sociedade Brasileira de Nefrologia. Censo geral. http://www.sbn.org.br/censosanteriores.asp (acessado em Fev/2008).

30. Lôbo MCSG, Bello VAO. Reabilitação profissional pós-transplante renal. J Bras Nefrol 2007; XXIX: 29-32. 
31. Peres LAB, Ann HK, Camargo MTA, Rohde NRS, Matsuo T, Uscocovich VFM, et al. Análise da sobrevida de enxertos e receptores de 188 transplantes renais realizados na cidade de Cascavel, PR. J Bras Nefrol 2003; XXV:133-41.

32. Manley HJ. Disease progression and the application of evidence-based treatment guidelines diagnose it early: a case for screening and appropriate management. J Manag Care Pharm 2007; 13(9 Suppl D):S6-12.
33. Vianna SM, Nunes A, Góes G, Silva JR, Santos RJM. Projeto Economia da Saúde. Atenção de alta complexidade no SUS: desigualdades no acesso e no financiamento. v. 1. http://getinternet.ipea.gov. $\mathrm{br} /$ economiadasaude/adm/arquivos/destaque/ alta_complexidade.pdf (acessado em Jan/2008).

Recebido em 05/Nov/2009

Versão final reapresentada em 26/Jul/2010 Aprovado em 26/Out/2010 